\title{
Characterization of nuclear pore complex targeting domains in Pom152 in Saccharomyces cerevisiae
}

\author{
Jacqueline T. Brown*, Alexandra J. Haraczy, Christopher M. Wilhelm§ and Kenneth D. Belangerף
}

\begin{abstract}
Pom152 is a transmembrane protein within the nuclear pore complex (NPC) of fungi that is important for NPC assembly and structure. Pom152 is comprised of a short amino-terminal region that remains on the cytosolic side of the nuclear envelope (NE) and interacts with NPC proteins, a transmembrane domain, and a large, glycosylated carboxy-terminal domain within the NE lumen. Here we show that the $\mathrm{N}$-terminal 200 amino acids of Pom152 that include only the aminoterminal and transmembrane regions are sufficient for localization to the NPC. Full-length, glycosylation-deficient, and truncated Pom152-GFP chimeras expressed in cells containing endogenous Pom152 localize to both NPCs and cortical endoplasmic reticulum (ER). Expression of Pom152-GFP fusions in pom1524 cells results in detectable localization at only the NE by full-length and aminoterminal Pom152-GFP fusions, but continued retention at both the NE and ER for a chimera lacking just the carboxy-terminal 377 amino acids. Neither deletion of Pom152 nor its carboxy-terminal glycosylation sites altered the nuclear protein export rate of an Msn5/Kap142 protein cargo. These data narrow the Pom152 region sufficient for NPC localization and provide evidence that alterations in other domains may impact Pom152 targeting or affinity for the NPC.
\end{abstract}

KEY WORDS: Pom152, Nuclear envelope, Nuclear pore complex, Nucleoporin

\section{INTRODUCTION}

Nuclear pore complexes (NPCs) are large, aqueous, proteinaceous channels that perforate the nuclear envelope of eukaryotic cells and regulate communication and transport between the nucleoplasm and cytoplasm (Beck and Hurt, 2017; Knockenhauer and Schwartz, 2016; Tran and Wente, 2006). In the yeast Saccharomyces cerevisiae, each NPC is composed of about 30 different proteins termed nucleoporins (Nups). Each Nup is present in multiples of eight copies within each NPC, resulting in the eightfold symmetry of the nuclear pores (Alber et al., 2007a; Kim et al., 2018; Mi et al., 2015; Rajoo et al., 2018). Nups can be categorized by grouping them based on their function and characteristic functional domains:

\footnotetext{
Department of Biology, Colgate University, Hamilton, NY 13346, USA.

*Present address: Winship Cancer Institute of Emory University, Atlanta, GA, USA

†Present address: University of Michigan Medical School, Ann Arbor, MI, USA.

§present address: Allen Institute for Artificial Intelligence, Seattle, WA, USA.

"Author for correspondence (kbelanger@colgate.edu)

(D) J.T.B., 0000-0002-1885-0906; A.J.H., 0000-0002-9065-7158; K.D.B., 0000 0002-7181-0188
}

This is an Open Access article distributed under the terms of the Creative Commons Attribution License (https://creativecommons.org/licenses/by/4.0), which permits unrestricted use, distribution and reproduction in any medium provided that the original work is properly attributed.

Received 10 November 2020; Accepted 20 September 2021
FG-Nups, non-FG-Nups, and pore membrane Nups. FG-Nups facilitate substrate translocation across the NPC via hydrophobic regions containing phenylalanine-glycine (FG) repeat sequences by forming a diffusion barrier within the pore that selectively allows passage of nuclear transport factors (NTFs) and their cargoes (Onischenko and Weis, 2011; Rout et al., 2000). Non-FG Nups form the structural scaffold with which FG-Nups associate within the NPC (Kim et al., 2018; Kosinski et al., 2016; Lin et al., 2016). The pore membrane Nups (POMs) span the pore membrane, the lipid bilayer within the NPC that connects the inner and outer nuclear membranes. In yeast, the pore membrane Nups are Pom152, Pom33, Pom34, and Ndc1, and each is involved in both assembly of nascent NPCs and maintenance of the structural organization of each NPC via association with each other and with non-FG Nups within the pore.

Pom152 is a type II transmembrane protein with an aminoterminal cytosolic domain, a single transmembrane (TM) domain spanning residues 176-195, and a carboxy-terminal lumenal domain that comprises most of the mass of the 1337 amino acid polypeptide (Tcheperegine et al., 1999; Wozniak et al., 1994). The carboxy-terminal domain self-oligomerizes within the NE lumen with eightfold symmetry and, together with Pom34 and Ndc1, forms the membrane ring complex at the core of each NPC (Kim et al., 2018; Upla et al., 2017; Yewdell et al., 2011). The C-terminal domain contains at least four N-linked glycosylation sites, although the function of this glycosylation remains unclear (Belanger et al., 2005; Wozniak et al., 1994; Yewdell et al., 2011). The aminoterminal, cytosolic Pom152 domain associates with non-FG Nups to form the scaffold around the equator of the NPC that provides the structure on which the FG-Nups assemble (Alber et al., 2007b; Kim et al., 2018). Evidence suggests that Pom 152 is present in either eight (Mi et al., 2015; Rajoo et al., 2018) or 16 (Alber et al., 2007a; Kim et al., 2018; Rout et al., 2000) copies per NPC.

Here we further examine the Pom152 sorting determinant responsible for localization at the yeast NPC and investigate the role of Pom152 in NPC function. We observe that the aminoterminal 200 amino acids of Pom152 completely lacking any lumenal domain are sufficient for targeting to NPCs. Expression of GFP-tagged versions of Pom 152 result in both nuclear envelope and cortical endoplasmic reticulum localization in cells expressing endogenous Pom152. Eliminating the expression of wild-type Pom152 decreases cortical ER localization of all Pom152-GFP chimeras tested except for a truncation lacking 377 amino acids from the carboxy-terminus. Pom152 mutants completely lacking the carboxy-terminus in conjunction with mutations of select residues within or adjacent to the transmembrane sequence showed localization comparable to wild-type Pom152, suggesting that at least these portions of the amino-terminal and TM domains are not required for Pom152 direction to the NPC. We also observe that deletion of POM152 or removal of its lumenal glycosylation sites does not significantly alter the kinetics of Msn5-mediated nuclear 
protein export from yeast nuclei, consistent with observations that pom152 mutants do not have nuclear protein import or mRNA export defects (Belanger et al., 2005; Madrid et al., 2006). These data add to our understanding of Pom152 domain structure and function within the NPC and targeting to NPCs.

\section{RESULTS}

The amino-terminal and transmembrane domains together are sufficient for localization of Pom152 to the yeast NPC

In order to further define the region of Pom152 sufficient for anchoring at the NPC, Pom152-GFP chimeras containing either

A.

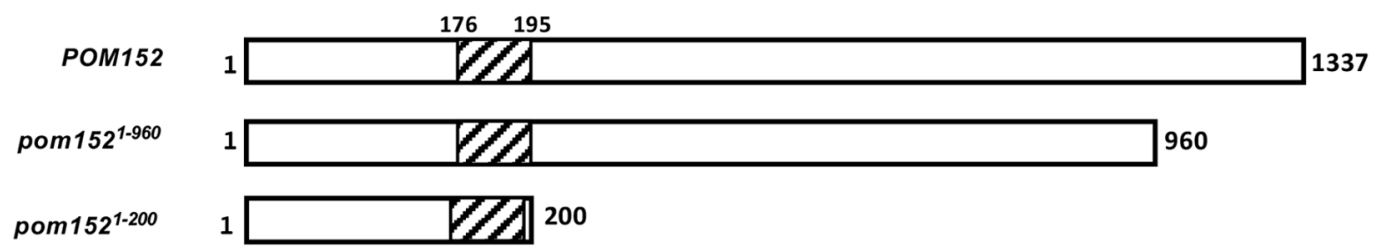

B.

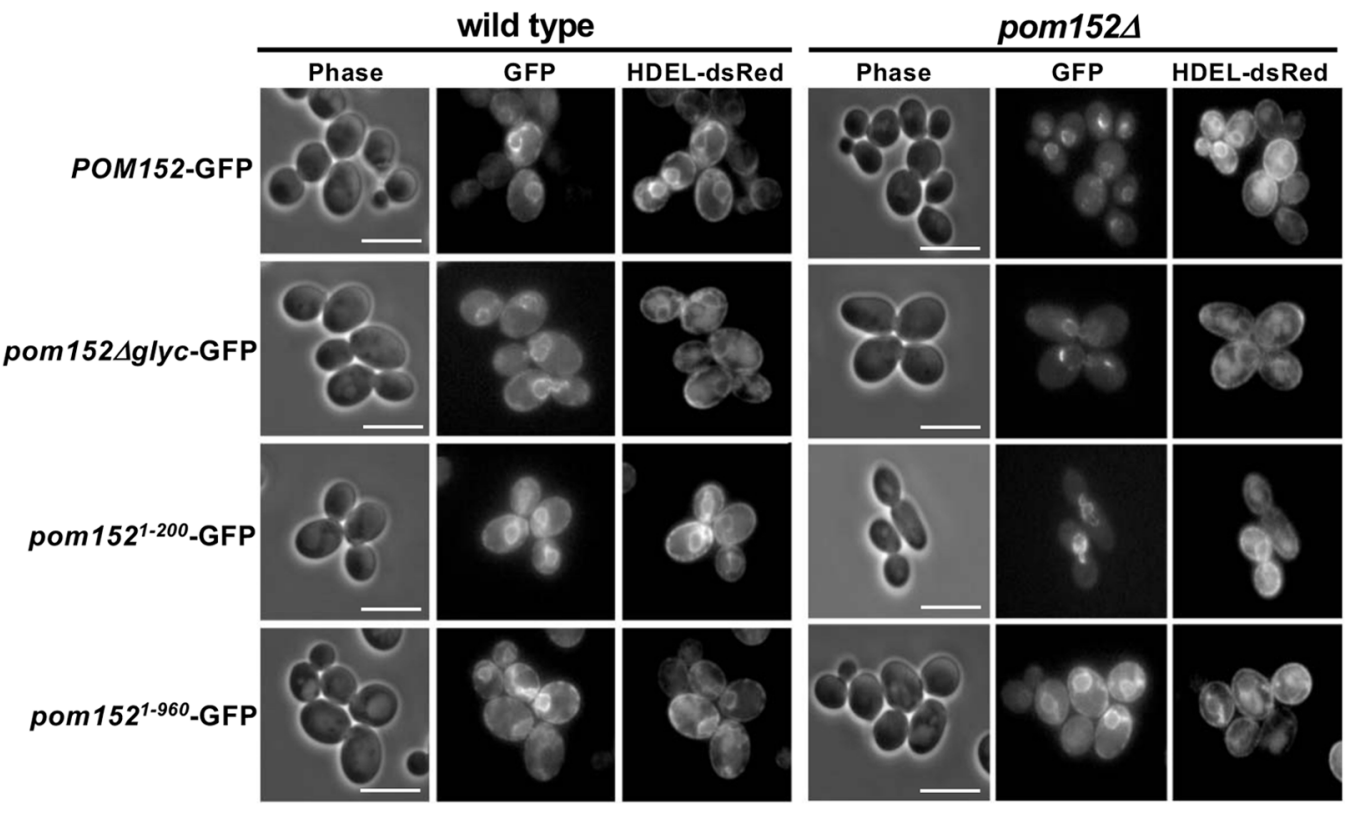

C.
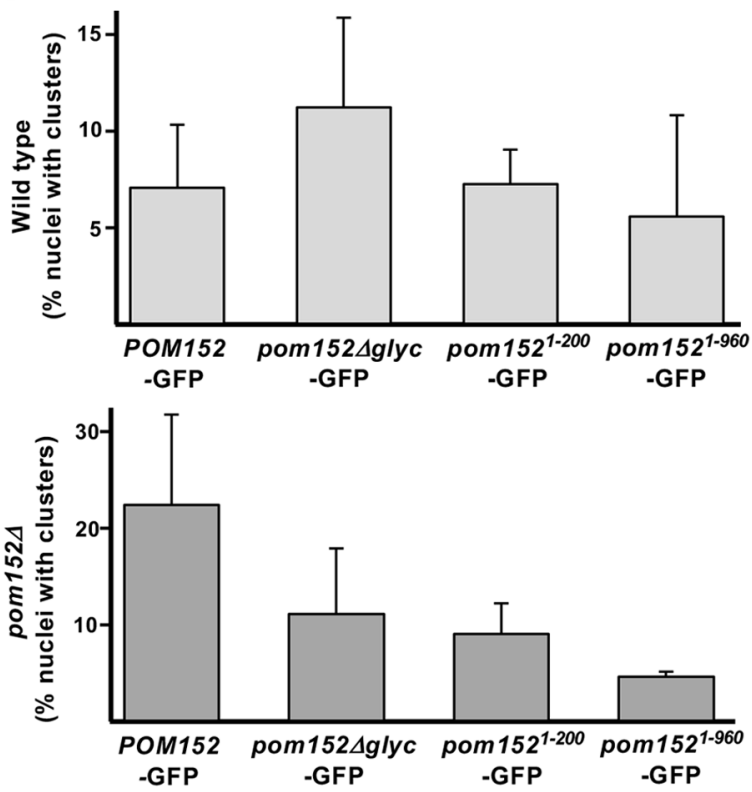

D.

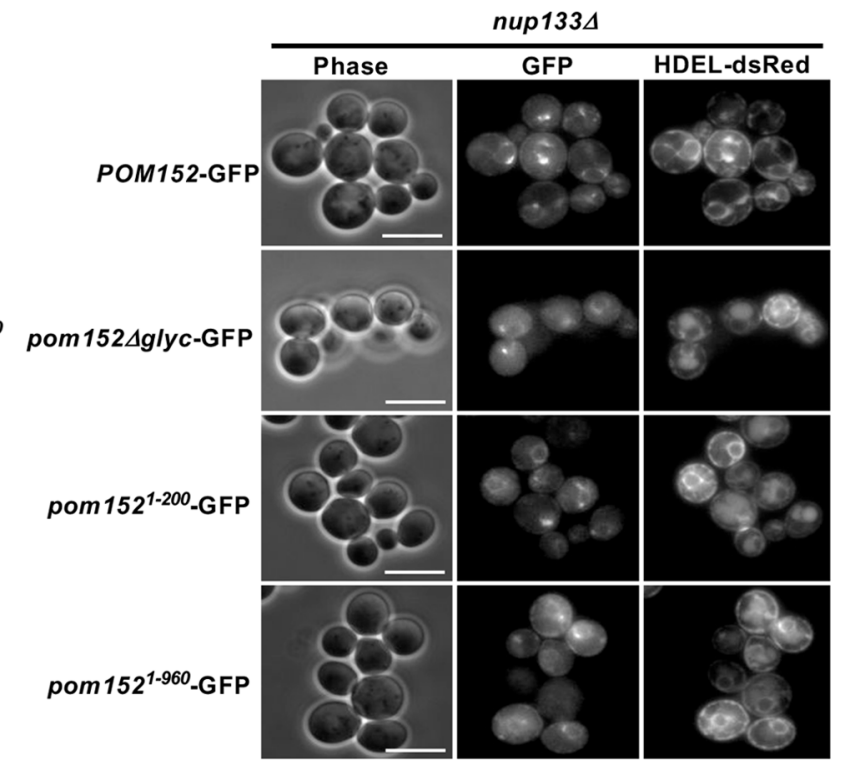

Fig. 1. See next page for legend. 
Fig. 1. Removal or deglycosylation of the Pom152 carboxy-terminal, lumenal domain does not alter localization at the NPCs, while truncation of a portion of the lumenal domain results in increased ER Iocalization. (A) Cartoon schematic of Pom152 carboxy-terminal truncations

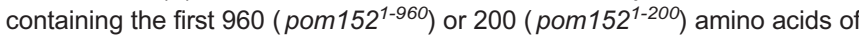
Pom152 expressed as chimeras with GFP. (B) Wt POM152-GFP, a mutant lacking four glycosylation sites from the carboxy-terminal, lumenal domain (pom152 $2^{\left.4 g / y c_{-}-G F P\right)}$, and C-terminal truncations pom152 ${ }^{1-200}$-GFP and pom152 ${ }^{1-960}$-GFP were expressed in wt yeast and yeast lacking endogenous POM152 ( pom1524). Cells were co-transformed with a plasmid expressing the endoplasmic reticulum marker HDEL-dsRed (Madrid et al., 2006) and observed via phase-contrast and fluorescence microscopy. pom152-GFP mutants localize to both the nuclear envelope and ER in cells expressing endogenous POM152, while only the Pom152 $2^{1-960}$-GFP retains ER localization in pom152 15 cells. Scale bars: $5 \mu \mathrm{m}$. (C) Quantitative analyses of nuclear envelope clustering of each Pom152-GFP fusion reveal no significant differences in localization in either wt [top panel; one-way ANOVA: $F(3,16)=1.55, P=0.24$ ] and pom152 [bottom panel; one-way ANOVA: $F(3,8)=1.36, P=0.32]$ yeast. (D) Expression of Pom152-,

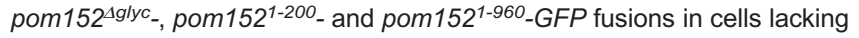
Nup133 (nup1334) results in clustered foci of fluorescence indicating NPC localization of all Pom152-GFP chimeras tested. Scale bars: $5 \mu \mathrm{m}$.

wild-type (wt) POM152 (Pom152-GFP), the amino-terminal and TM sequences exclusively (residues $1-200$; pom152 1 -200_GFP), a truncation of the carboxy-terminal 377 amino acids (residues 1-960; pom152 $1-960$ GFP), or POM152 lacking glycosylation sites at residues 280, 398, 569, and 1099 in the C-terminal lumenal domain (pom152 $2^{\Delta \text { glyc }}$-GFP; Belanger et al., 2005) were transformed into wt yeast (Fig. 1A). These Pom152-GFP-expressing plasmids were co-transformed with a plasmid encoding the endoplasmic reticulum (ER) targeting sequence HDEL fused to the red fluorescent protein DsRed (HDEL-DsRed; Madrid et al., 2006) and observed by phase contrast and fluorescence microscopy. When expressed in wt yeast, all Pom152-GFP chimeras localized to the NE and likely to the NPCs as evidenced by the characteristic punctate GFP-fluorescence pattern around the nuclear rim (Fig. 1B, left panel). Each Pom152 fusion also exhibited some co-localization with HDEL-DsRed fluorescence, indicating targeting to or retention within the cortical ER. To test if intracellular localization of the Pom152-GFP chimeras was affected by the presence of endogenous wt Pom152, we cotransformed plasmids expressing the same four Pom152-GFP chimeras and the HDEL-DsRed ER marker into yeast lacking POM152 (pom1524) and observed transformed cells by phase contrast and fluorescence microscopy. Localization of Pom152 ${ }^{\mathrm{wt}}$, pom $152^{\Delta \mathrm{glyc}}$ and pom $152^{1-200}$ to the cortical ER was reduced to the point of no longer being detectable, but pom152 $2^{1-960}$ retained significant localization to the cortical ER (Fig. 1B, right panel). These observations suggest that no portion of the lumenal carboxyterminus of Pom152 is required for NPC localization, but that alterations in the carboxy-terminal domain may alter NPC affinity or targeting relative to wt.

Some cells expressing each Pom152-GFP fusion exhibited what appear to be clusters of more intense fluorescence in regions of the nuclear envelope, suggesting localization of the tagged Pom152 protein to a subset of NPCs or an NPC clustering phenotype resulting from Pom152-GFP expression (see Fig. 1B). In order to determine whether the frequency of this 'clustering' phenotype varied among Pom152 mutants, we quantified the percentage of cells expressing each Pom152-GFP construct that showed more intense fluorescence in small regions of the nuclear envelope (Fig. 1C). A one-way ANOVA was conducted to determine if the frequency of Pom152 clustering at the nuclear envelope was different between the Pom152-GFP constructs tested in wt and in pom $152 \Delta$ yeast.
In the wt yeast background, Pom152-GFP fusions examined for intracellular localization were Pom $152^{\mathrm{wt}}$-GFP, Pom $152^{\Delta \mathrm{Glyc}}$-GFP, Pom $152^{1-200}$-GFP, and Pom152 $1-960$-GFP. The data are expressed as the mean percentage of cells with fluorescent clustering at the nuclear envelope \pm the standard error of the mean, based on a minimum of three independent analyses of over 50 cells each for each strain. The mean percent clustering of Pom $152^{\mathrm{wt}}$-GFP was $7.1 \pm 3.3 \%$, Pom $152^{\Delta \mathrm{Glyc}}$-GFP was $11.3 \pm 4.7 \%$, Pom $152^{1-200}$-GFP was $7.3 \pm 1.8 \%$, and Pom $152^{1-960}$-GFP was $5.6 \pm 5.3 \%$. A one-way ANOVA was performed and the differences between the log transformed average of cells exhibiting nuclear clustering were not statistically significant $[F(3,16)=1.55, P=0.24]$ between these Pom152-GFP constructs in wt yeast. In pom152 1 yeast, a similar ANOVA analysis was performed on the nuclear envelope localization of the same four Pom152-GFP constructs. This analysis revealed no statistically significant differences in fluorescence localization among the Pom152-GFP constructs $[F(3,8)=1.36, P=0.32]$. The mean percent clustering of Pom $152^{\mathrm{wt}}$ GFP was $22.4 \pm 9.4 \%$, Pom $152^{\Delta \mathrm{Glyc}}$-GFP was $11.1 \pm 6.8 \%$, Pom $152^{1-}$ ${ }^{200}$-GFP was $9.0 \pm 3.2 \%$, and Pom $152^{1-960}$-GFP was $4.6 \pm 0.5 \%$, based on a minimum of two independent analyses of greater than 35 cells each.

While all strains exhibited some cells in which various Pom152GFP fusions appear to cluster in small regions of the NE (Fig. 1B), based on our quantitative analysis of these data, none of the pom152-GFP mutants cluster within the nuclear envelope at a frequency that is significantly different from full-length Pom152GFP in either wt or pom152 1 yeast.

The partial localization of pom $152^{1-960}$ to the endoplasmic reticulum in both wt and pom $152 \Delta$ yeast raised the possibility that the perceived NPC localization of the Pom152-GFP fusions might be due to localization to the nuclear envelope as a consequence of this membranous structure being contiguous with the cortical ER. To confirm NPC localization, we expressed the same four Pom152GFP chimeras in nup $133 \Delta$ cells that exhibit a temperature-sensitive, NPC clustering phenotype (Belgareh and Doye, 1997) and contain the HDEL-dsRed ER marker. Phase contrast and fluorescence microscopy after incubation at the restrictive temperature showed all four Pom152-GFP fusions exhibiting clustered fluorescence around one side of the nuclear rim, characteristic of NPC localization in nup $133 \Delta$ cells (Fig. 1D). A small amount of each Pom152-GFP remained in the cortical endoplasmic reticulum around the periphery of the cell. These observations suggest that these Pom152-GFP chimeras are localized to NPCs and provide evidence that the first 200 amino acids of Pom152 completely lacking any lumenal sequence are sufficient for NPC localization.

\section{Alteration of specific residues within and adjacent to the Pom152 transmembrane sequence does not affect localization to the yeast NPC}

Metazoan NPCs contain a single-pass transmembrane protein named gp210/Nup210 that exhibits some similarities to Pom152, including involvement in NPCs assembly and structural organization (Stavru et al., 2006). Nup210 also has a large lumenal domain and a smaller cytosolic region like Pom152, but the topology of the protein is switched such that the $\mathrm{N}$-terminus is in the NE lumen and the C-terminus is cytosolic (Greber et al., 1990). Interestingly, the transmembrane domain alone of Nup210 is sufficient for targeting to the NPC, as is a 20 amino acid region of the cytosolic domain of the protein (Wozniak and Blobel, 1992). Because the Nup210 transmembrane sequence and cytosolic domain each contain sorting determinants sufficient for 
A.

S. Cerevisiae
Pom152
Pom158A,F159A,F160A
S. Paradoxus
S. Eubayanus
S. Pastorianus
N. Castellii
Z. mellis
T. Delbrueckii
N. Dairenensis
Z. bailii
E. Cymbalariae
K. dobzhanskii
K. Naganishii
T. Phaffii
L. thermotolerans
K. lactis
E. Gossypii
A. aceri
V. Polyspora
S. Ludwigii
C. Glabrata
S. pombe

FNFISKYFI IDSFFLYVLPSFNI PRLTFKPWVVYLQILAMLLLNIF ISSDHEFV FNFISKYFI IDAAALYVLPSFNI PRLTFKPWVVYLQILAMLLLNIF I SSDHEFV FNFISKYFIIDSFFLYVLPSFNIPRLTFKPWVVLLLILAMLLLNIF ISSDHEFV FNFISKYFIMDSLFLYVLPSFNI PRLTFKPWMVYLQILGMILLN IF ISSDHEFV FNFISKYFIVDSLFLYILPSFNI PRLTFKPWMVYLQVLTMTALSVF ISSDHEFV FNFISKYFIVDSLFLYILPSFNIPRLTFKPWMVYLQVLTMTALSVF ISSDHEFV FNFVTKYIVIDSLFF F ILPNFNI PKLTFRKWIVYLQVI IMAS ITIFLSTEHEF I FNFVSKYVVVDAMFLYFLPSFKIPKLTFOPWVVLLQILCMWVITFFISTEHDF INFI IKYFLIDSMFLYFLPTFKIPKLCFKSWAVFVQLVVMTLLTILISSEQEFV FNFISKYLIIDSMFFYLLPTFNIPKLTFTKWIVI IQIA IMSITTIVLSQEHNF I FNFISKYVVIDAMFLYFLPSFKIPKLTFPPWVVCLQTVFMWGFTLFVSTEHDFT FNFISKYAIIDSLFLYFLPSFKI PKLCFKPFI IFAOILVMIALTVLLSNDKNF LNFISKYFVIDSLFFYCLPTFKIPKLKFKPIIVFFQILSFVALTVFISSEQSF FNFITKYLLLDSMFWYFLPIFRIPRLTSKPWLVGFQIVATAATTIFLSTNHQFV FGFLLKYLIIDSCYFYFLPSFNIPTLTFKTWTITLQIMSIFMFNIFLTNDHEWI FNFISKYLVIDSLFLYFLPGLKIPKLTFRPVAVLLQIMAVTATTVFLSNEQNF LNFISKYLVIDSLFFYCLPTFKI PKLKFKPLIVFLQI ISSATLTLF ISSEQ FNFISKYAIVDSLFLYFLPSFKIPKLRFRPIVTFLQILLAVALTILLSND FNFISKYAIVDSLFLYFLPSFKIPKLRFRPIVTFLQIMLAVALTILLSND TTFVSKYLLIDSLFFYVLSTFKIPKLVFKSWVILVQIVLMSLITIGISSHTDFV TGFL IKYALLDSLFWYSLPAFNIPKLIFKPVVTY IFIFLS ILFNSLIS SDK FNFLFKYLIVDAAFFFTLPVLNIPKLTFPTWVVISQVLLVTSFNIALCNNKTTV

B.

POM152
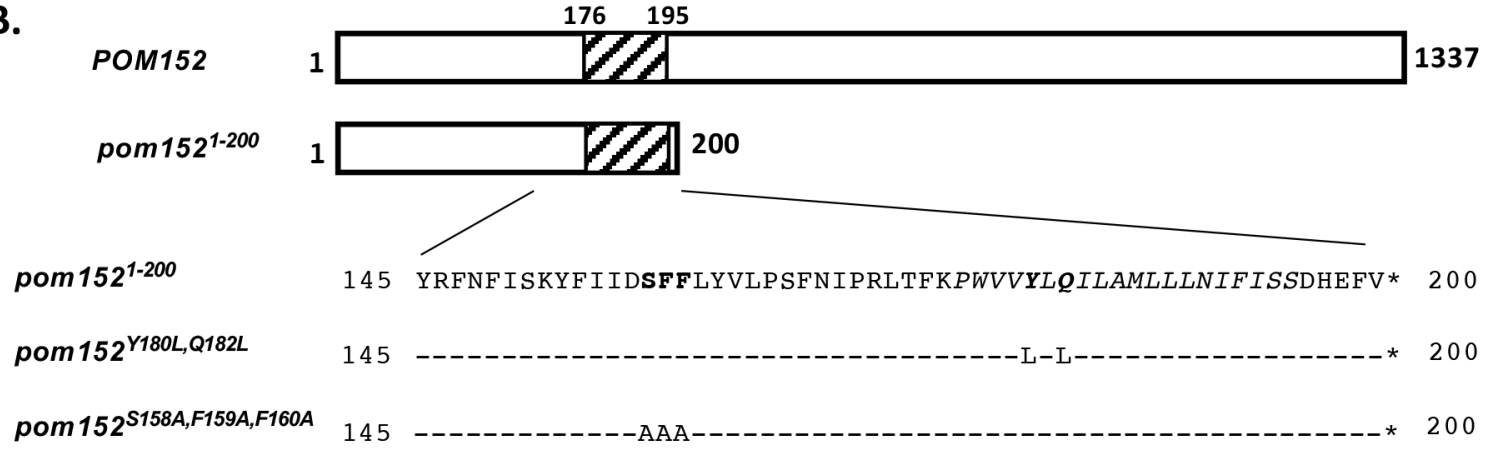

C.

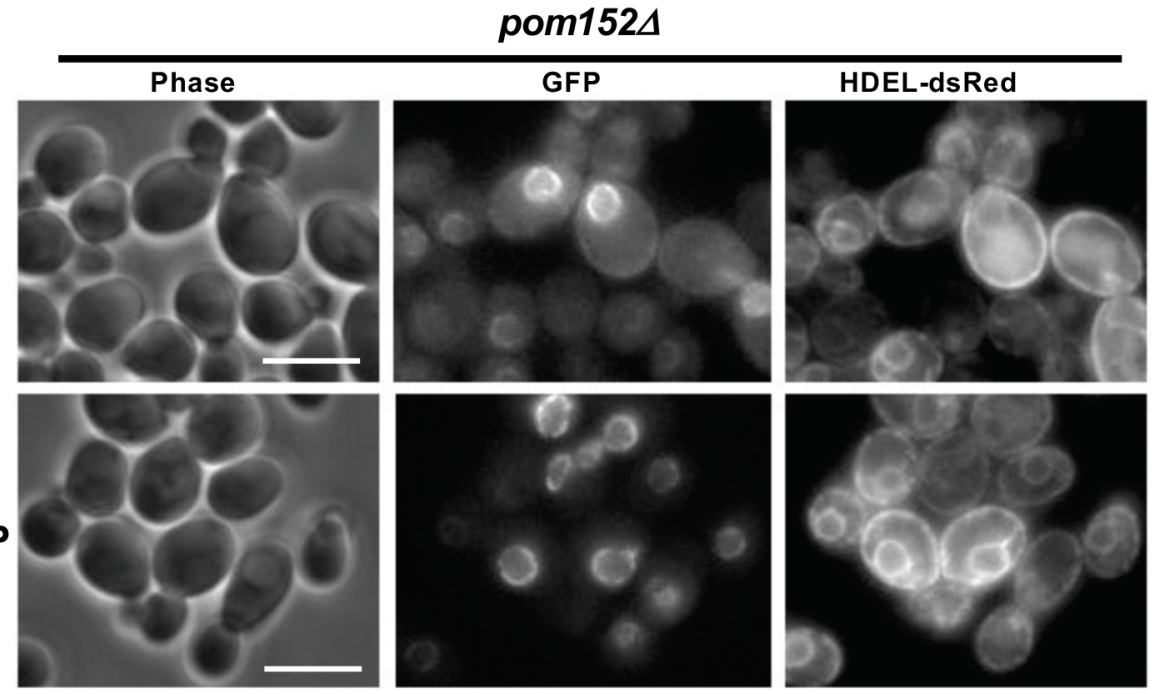

pom152S158A,F159A,F160A_GFP

pom152 ${ }^{Y 180 L, Q 182 L-G F P}$

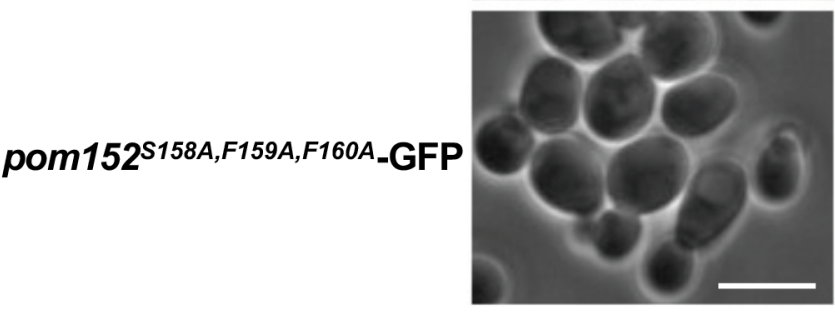

Fig. 2. See next page for legend.

localization to the mammalian NPC (Wozniak and Blobel, 1992), we sought to identify sequences that function similarly in and adjacent to the Pom152 transmembrane and cytosolic domains.
Although the amino acid sequence of Pom152 does not align with Nup210 in this region, a query of BLAST (Altschul et al., 1990) using the first 200 amino acid residues from Pom152 reveals 
Fig. 2. Targeted mutations within or flanking the transmembrane domain of Pom152 do not affect localization to the nuclear envelope. (A) Alignment of amino acid residues 147-200 from S. cerevisiae Pom152 with conserved regions of Pom152 from other fungi. Yellow highlights indicate identities with $S$. cerevisiae. Green highlights depict residues altered in pom152 $158 A, F 159 A, F 160 A$-GFP and blue highlights depict those

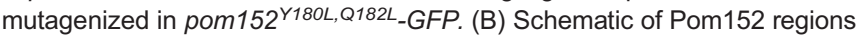
used to make POM152-GFP and pom152 ${ }^{1-200}$-GFP, pom152 ${ }^{\text {Y180L,Q182L }-G F P}$ and pom152 ${ }^{S 158 A, F 159 A, F 160 A}$ _GFP chimeras. (C) Phase-contrast (phase) and fluorescence imaging of yeast deleted for POM152 (pom1524) COexpressing pom152 ${ }^{1-200}$-GFP mutants with the ER marker HDEL-dsRed. The pom152 ${ }^{\text {Y180L,Q182L }}$ GFP and pom152 ${ }^{\text {S158A,F159A,F160A_GFP fusions both }}$ localize to the nuclear envelope. Scale bars: $5 \mu \mathrm{m}$

significant homology across fungal organisms, including some residues that are conserved across nearly all species examined (Fig. 2A). Among these conserved residues are a serine at residue 158 (S158) in S. cerevisiae Pom152, a phenylalanine at residue 160 (F160), a glutamine at residue 182 (Q182). We used site-directed mutagenesis to create two mutant pom 152 1-200-GFP fusions (Fig. 2B): one in which Tyr180 and Gln182 are both replaced by Leu (pom152 ${ }^{\mathrm{Y} 180 \mathrm{~L}, \mathrm{Q} 182 \mathrm{~L}}$-GFP) and another in which Ser158Phe159-Phe160 are changed to Ala residues (pom152 $158 \mathrm{~A}, \mathrm{~F} 159 \mathrm{~A}$, F160A_GFP). Yeast strains deleted for POM152 (pom152A, Fig. 2C) expressing each transmembrane domain mutant and HDEL-dsRed were observed by phase contrast and fluorescence microscopy. Both pom152 transmembrane mutants localized to the NPC with no observable difference from wt Pom152-GFP or the pom152 $2^{1-200}$ GFP truncation (compare Figs 1B and 2C), suggesting that these specific residues of the TM sequence or its flanking cytosolic sequence are not required for Pom152 targeting to or anchoring at the pore membrane domain.

\section{Pom152 and Pom152 glycosylation are not essential for Crz1 nuclear protein export}

Deletions of POM152 or elimination of four glycosylation sites from the Pom152 lumenal domain do not detectably alter nuclear protein import kinetics or mRNA nuclear export (Belanger et al., 2005; Madrid et al., 2006). To examine whether a loss of Pom 152 or its glycosylation altered the rate of facilitated nuclear export of a protein that had accumulated in the nucleus, we performed kinetic assays for Msn5/Kap142-mediated nuclear protein export on cells that had accumulated the transcription factor $\mathrm{Crz} 1$ in the nucleus and were subsequently induced to export the protein to the cytoplasm. Live-cell fluorescence microscopy showed rapid export of Crzl from yeast nuclei with a disappearance of detectable nuclear Crz1GFP over a similar time span in wt, pom1524, and pom152 1 glyc cells (Fig. 3). To better understand the relationship between the Crz1 nuclear export rates for each pair of yeast strains tested, a robust linear regression was run. The percent nuclear data for each genotype was averaged for each time point, log-transformed, and the slope of the resulting line for each genotype was obtained using the Theil-Sen method (Sen, 1968). We then used Mann-Whitney $U$-test to compare the slope differences between each pair of yeast strains, and adjusted the $P$-values for multiple testing using BenjaminiHochberg method (Benjamini and Hochberg, 1995). The only significant differences observed were between msn5 $\Delta$ and the wt $\left(P=2.63 \times 10^{-9}\right)$, pom 152::KAN $\left(P=2.49 \times 10^{-2}\right)$, pom152::NAT $\left(P=4.03 \times 10^{-8}\right)$, and pom 1524glyc $\left(P=1.57 \times 10^{-10}\right)$ strains. There were no significant differences detected between wt and the pom152 mutant strains tested $(P>0.01)$. These data suggest that neither a full deletion of POM152 nor removal of the lumenal glycosylation sites significantly alters Msn5-mediated nuclear protein export.

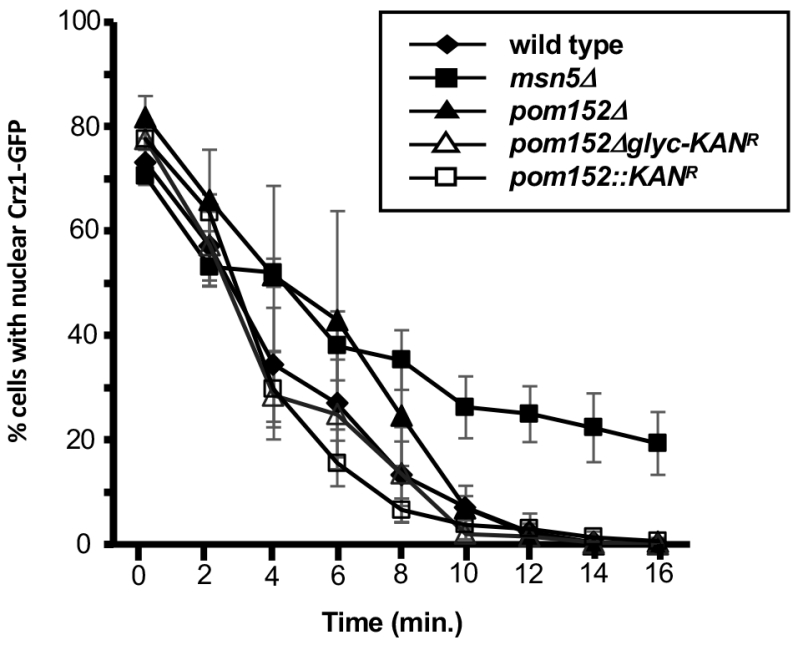

Fig. 3. Pom152 deletion or removal of glycosylation sites does not alter Msn5-mediated nuclear protein export. Wt, pom152 , and pom152 1 glyc cells expressing Crz1-GFP were allowed to accumulate Crz1-GFP in the nucleus in the presence of calcium and the percentage of cells with distinct nuclear fluorescence was recorded. Crz1 nuclear export was then induced by the addition of FK506. Data points indicate the percentage of cells with nuclear fluorescence for each strain. Error bars represent standard error of the mean. Significant differences in export rate were observed between $m s n 5 \Delta$ and wt $\left(P=2.63 \times 10^{-9}\right)$, pom152::KAN $\left(P=2.49 \times 10^{-2}\right)$, pom152::NATr $\left(P=4.03 \times 10^{-8}\right)$, and pom $152 \Delta$ glyc $\left(P=1.57 \times 10^{-10}\right)$ strains. Significant differences were not detected between wt and the pom152 mutants tested $(P>0.01)$, suggesting that deletion of $P O M 152$ or removal of its carboxyterminal glycosylation does not affect Msn5-mediated nuclear export kinetics.

\section{DISCUSSION}

In this study, we have presented evidence that amino-terminal amino acid residues 1-200 of the pore membrane nucleoporin Pom152, including only the cytosolic and transmembrane regions, are sufficient for targeting the protein to the nuclear pore complex in Saccharomyces cerevisiae. Carboxy-terminal residues 201-1337 of Pom152 localized within the NE lumen are not necessary for NPC localization, but removal of a portion of the carboxy-terminal domain may affect Pom152 targeting to and/or affinity for the NPC, as evidenced by continued cortical ER localization of pom $152^{1-960}$ GFP, even after deletion of endogenous Pom152. Deletion of POM152 or removal of its carboxy-terminal glycosylation sites do not alter the rate of nuclear export of the shuttling transcription factor Crz1.

\section{Pom152-GFP chimeras localize to both NPCs and cortical ER} in the presence of endogenous Pom152

All of the Pom152-GFP chimeric proteins tested localize to both the NE and the cortical ER in wt yeast expressing endogenous POM152. These data may suggest that co-expression of the recombinant Pom152-GFP fusions and endogenous Pom152 results in saturation of Pom152 sites within the NPC and that excess Pom152 not integrated into the nuclear pores is retained throughout the ER membrane. Alternatively, the observation of some Pom152-GFP in the ER membrane may indicate a lower affinity of the recombinant protein than endogenous Pom 152 for the $\mathrm{NPC}$, although the presence of some recombinant protein at the $\mathrm{NE}$ indicates that any reduction in affinity is not so great that Pom152-GFP chimeras are essentially excluded from NPCs. The observation that expression of Pom152-GFP, pom152 $\Delta$ glyc-GFP and pom $152^{1-200}$-GFP in cells lacking endogenous Pom152 results 
in NE envelope localization but undetectable fluorescence in the ER suggests that the affinity of these recombinant proteins for the NPC is sufficiently high for efficient targeting and/or retention. It has been shown previously that the amino-terminal region of Pom 152 binds directly to the pore membrane Nups Pom34 and Ndc1 and to the non-FG nucleoporin Nup170 to link the membrane ring complex at the pore membrane to the inner ring complex that provides the inner scaffolding of the NPC (Kim et al., 2018; Makio et al., 2009; Onischenko et al., 2009) and this association likely mediates or stabilizes the Pom152 amino-terminal region at the NPC. Onischenko et al. (2009) observed that removal of the amino-terminal 170 amino acids of Pom152 alters NPC targeting, with the resulting truncation present at both the NPC and other intracellular membranes, likely predominantly cortical ER, suggesting that amino acids both within and downstream of the first 170 residues of Pom152 may be involved in NPC targeting and retention.

\section{Pom152 ${ }^{1-960}$-GFP is present in cortical ER even in the absence of Pom152}

Unlike the other Pom152-GFP proteins we tested, pom152 1-960 GFP retains fluorescence in the ER as well as the NPC in pom152 $\Delta$ yeast, suggesting that removal of the carboxy-terminal 377 amino acids from Pom152 may reduce NPC targeting or retention to the extent that a detectable fraction of the expressed protein is present in the ER. Alternatively, this truncated chimera may be more stable or may be overexpressed, resulting in excess pom $152^{1-960}$-GFP accumulating in the ER. The carboxy-terminal region of Pom152 extends from residues 200-1337 and is composed of eight or more immunoglobulin (Ig)-like folds that interact within the NE lumen and self-assemble head-to-tail and antiparallel to form the lumenal membrane ring at the equator of each NPC (Hao et al., 2018; Upla et al., 2017). Pom $152^{1-960}$-GFP entirely lacks the carboxy-terminal three Ig-like folds and truncates a portion of the fourth. The Pom152 lumenal domain mediates Pom152 self-assembly within the membrane ring and all of the Ig-like folds are proposed to be involved in the anti-parallel association of juxtaposed Pom152 polypeptides (Hao et al., 2018; Kim et al., 2018; Upla et al., 2017; Yewdell et al., 2011). Thus, it is not unexpected that a loss of a portion of the Pom152 lumenal domain would alter NPC targeting of the protein.

\section{Mutations in the Pom152 transmembrane domain do not alter localization}

Interestingly, the TM region alone of gp210/Nup210, the metazoan pore membrane NPC protein most structurally and functionally similar to Pom152, is sufficient for NPC localization (Wozniak and Blobel, 1992). Our limited mutagenesis of transmembrane (pom152 $\mathrm{Y} 180 \mathrm{~L}, \mathrm{Q} 182 \mathrm{~L}-\mathrm{GFP})$ and flanking aminoterminal ( pom152 $2^{\mathrm{S} 158 \mathrm{~A}, \mathrm{~F} 159 \mathrm{~A}, \mathrm{~F} 160 \mathrm{~A}}$-GFP) residues in pom152 $1-200_{-}$ GFP chimera did not reveal any changes in localization when expressed in wt and pom152 $\Delta$ cells and appeared to be targeted identically to the pattern observed for pom $152^{1-200}$-GFP. Pom152 and gp210/Nup210 both are large, single-pass pore membrane nucleoporins with a large lumenal domain and shorter cytoplasmic region (Greber et al., 1990) that are important for NPC assembly and structural organization (Stavru et al., 2006). However, they contain only limited sequence similarity and are oriented such that the lumenal domain is at the carboxy-terminus of Pom152, but at the amino-terminus of gp210/Nup210 (Greber et al., 1990; Wozniak et al., 1994). However, yeast Pom152 expressed in mammalian cells localizes to NPCs (Wozniak et al., 1994), suggesting at least some conservation of intracellular targeting mechanism may occur. The development of a more comprehensive library of mutants and truncations will be necessary to more narrowly identify NPC targeting regions in Pom152 and determine if these sequences have any similarity to the gp210/Nup210 NPC targeting regions.

\section{Deletion of Pom152 does not alter Crz1 nuclear export kinetics}

While previous work indicated that Pom 152 alterations do not affect nuclear protein import and mRNA export through the NPCs (Belanger et al., 2005; Madrid et al., 2006), an analysis of the impact of Pom152 deletion or deglycosylation on nuclear protein export has not been reported. Here we show, not unexpectedly, that

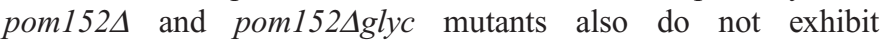
significantly slowed rates of Msn5-mediated Crz1-GFP export from the nucleus. Despite the roles for Pom152 in NPC assembly and organization (Madrid et al., 2006; Marelli et al., 2001; Onischenko et al., 2009; Yewdell et al., 2011), direct and indirect physical associations with other nucleoporins in the NPC (Alber et al., 2007a,b; Kim et al., 2018; Onischenko et al., 2009), and its synthetic genetic interactions with FG- and non-FG-NUP mutants (Belanger et al., 2005; Onischenko et al., 2009; Tcheperegine et al., 1999; Wozniak et al., 1994), to our knowledge deletions of POM152 alone have yet to exhibit a phenotype relating to nuclear transport, NPC structure or distribution, or NPC assembly. This observation is likely a result of partial functional redundancy with the pore membrane Nups Ndc1 and Pom34, together with which Pom152 forms a complex in the pore membrane ring and interacts with non-FG Nups to organize the inner ring scaffold of the NPC (Alber et al., 2007b; Kim et al., 2018; Onischenko et al., 2009). Additional work is necessary to identify the exact role of Pom 152 in NPC assembly and organization, the residues within Pom152 that mediate its targeting and function, and how a conserved protein performing these functions can be deleted without apparent nuclear transport, NPC structure, or cell growth or viability phenotypes.

\section{Conclusions}

In this study, we have generated a series of alterations in the $S$. cerevisiae pore membrane nucleoporin Pom 152 and have used these recombinant proteins to examine Pom152 targeting to NPCs. We observe that a Pom152-GFP chimera entirely lacking the carboxyterminal lumenal domain of the protein localizes to NPCs in a pattern indistinguishable from full length Pom152-GFP, suggesting that the targeting sequence is in the amino-terminal cytosolic and/or transmembrane regions. A shorter truncation of the lumenal domain increases retention in the cortical ER, raising the possibility that the self-assembling lumenal domain may also have a role in NPC targeting or retention. Pom152 alterations tested do not affect facilitated nuclear export of a shuttling transcription factor. In summary, the amino-terminal 200 amino acids of Pom152 target the protein to the NPCs in yeast.

\section{MATERIALS AND METHODS}

\section{Yeast strains, plasmids, and molecular techniques}

Yeast strains (Table 1) and plasmids (Table 2) used in this study are listed in the tables below. Introduction of DNA into yeast was performed by lithium acetate transformation (Gietz and Woods, 2006) and growth and selection were performed using established protocols. POM152-GFP (pKBB463), pom 152 1 glyc-GFP (Belanger et al., 2005; pKBB464), pom1521-960-GFP ( $\mathrm{pKBB} 521$ ), and pom152 ${ }^{1-200}$-GFP (pKBB520) fusions were generated by amplifying sGFP from $\mathrm{pJK} 19-1$ using PCR with PfuUltra ${ }^{\mathrm{TM}} \mathrm{HF}$ (Agilent Inc., Santa Clara, CA, USA) and primers that generated chimeric Pom152-sGFP 
Table 1. Yeast strains used in this study

\begin{tabular}{|c|c|c|}
\hline Strain & Genotype & Source \\
\hline BY4742 & MAT $\alpha$ his3 leu2 lys2 ura3 & Open Biosystems \\
\hline KBY776 & $M A T \alpha$ pom152 $K A N^{R}$ his3 leu2 lys2 ura3 & Open Biosystems \\
\hline KBY850 & 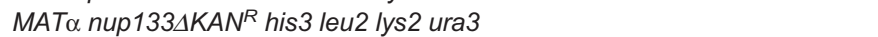 & Open Biosystems \\
\hline KBY893 & MAT $\alpha$ msn5 $4 K A N^{R}$ his3 leu2 lys2 ura3 & Open Biosystems \\
\hline KBY1292 & 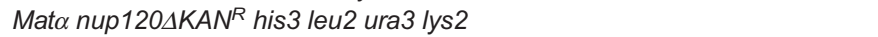 & Open Biosystems \\
\hline Y5563 & MAT $\alpha$ can14::MFA1pr-HIS3 lyp1 leu2 met15 ura3 his3 & (Tong and Boone, 2006) \\
\hline KBY1377 & MAT $\alpha$ can14::MFA1pr-HIS3 lyp1 leu2 met15 ura3 his3 pom152Aglyc-NAT ${ }^{R}$ & This study \\
\hline KBY1378 & 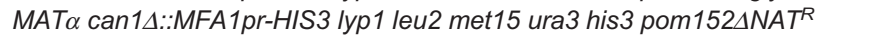 & This study \\
\hline
\end{tabular}

Table 2. Plasmids used in this study

\begin{tabular}{|c|c|c|}
\hline Plasmid name & Genotype & Source \\
\hline pRS315 & CEN LEU2 Amp $p^{R}$ & (Sikorski and Hieter, 1989) \\
\hline pJK19-1 & pET12a-sGFP & Pamela Silver \\
\hline pLMB127 & CEN URA3 3x-GFP-Crz1 Amp $p^{R}$ & (Boustany and Cyert, 2002) \\
\hline pKW1803 & YIP-Lac dsRed-HDEL NatMX & (Madrid et al., 2006) \\
\hline pPM1-HA & CEN LEU2 Amp ${ }^{R}$ POM152-HA & (Wozniak et al., 1994) \\
\hline pKBB382 & CEN LEU2 Amp ${ }^{R}$ pom152 1 glyc-HA & (Belanger et al., 2005) \\
\hline pKBB463 & CEN LEU2 Amp ${ }^{R}$ POM152-HA-sGFP & This study \\
\hline pKBB464 & CEN LEU2 Amp ${ }^{R}$ pom152 1 glyc-sGFP & This study \\
\hline pKBB520 & CEN LEU2 Amp $p^{R}$ pom $152^{1-200}-s G F P$ & This study \\
\hline pKBB521 & CEN LEU2 Amp ${ }^{R}$ pom152 $152^{1-960}$ s GFP & This study \\
\hline pKBB553 & CEN LEU2 Amp $p^{R}$ pom152 ${ }^{Y 180 L, Q 182 L}$-sGFP & This study \\
\hline pKBB556 & CEN LEU2 Amp ${ }^{R}$ pom152 ${ }^{S 158 A, F 159 A, F 160 A}$-sGFP & This study \\
\hline
\end{tabular}

PCR products for homologous recombination in yeast. For pKBB463 and pKBB464, primers KOL236 (5'- GAAATTACAGATGCTTATTGTTTTGCCAAAAATGATCTTTTTTTCAATAACGCTAGCAAAGGAGAAGAACTC-3') and KOL307 (5'- ATTTCTGTGGATGTTCAAAAGTCTGCTTTTAACACACCTCTATAGACCGTCCTTTCGGGCTTTGTTAGCAGCC-3') were used for amplification and the resulting PCR products were co-transformed into yeast strain BY4742 with either plasmid pPM1HA (Wozniak et al., 1994) and pKBB382 (Belanger et al., 2005) linearized with restriction endonuclease $P s h$ AI. Primers used to amplify sGFP from pJK19-1 were KOL407 (5'-AGAAGAAGTCTGTCAAGGGATGGAAGGTACGGTTGATTTGGCTCTATTTGGTTCTCCAGCTAGCAAAGGAGAAGAACTC-3') and KOL307 for pKBB521 and KOL406 (5'-CAGATTTTAGCTATGCTACTATTGAACATTTTCATATCAAGCGATCACGAGTTCGTTGCTAGCAAAGGAGAAGAACTC-3') and KOL307 for pKBB520, and PCR products were independently co-transformed into BY4742 with PshAI-linearized pPM1-HA. Cells containing recombinant plasmids were selected for on SD-Leu media and Pom152-sGFP fusions were confirmed by PCR and DNA sequencing. Site-directed mutagenesis with a Stratagene QuikChange Lightning Multi Site-Directed Mutagenesis Kit (Agilent, Inc., Santa Clara, CA, USA) was used to generate pom152 ${ }^{\text {Y180L,Q182L }}$-GFP (pKBB553) and pom152 ${ }^{\text {S1584,F159A,F160A }-G F P}$ (pKBB556) from pom152 $1-200-G F P$ (pKBB520) using mutagenic oligonucleotides KOL434 (5'- GCCATGGGTTGTTTTACTCCTGATTTTAGCTATGC-3') and KOL437 (5'- GTATTTCATTATAGATGCCGCCGCCCTGTATGTTTTACCATCC-3'), respectively, as per the manufacturer's instructions. Successful mutagenesis was confirmed by DNA sequencing.

\section{Fluorescence Microscopy}

Yeast strains (Table 1) were co-transformed with plasmids expressing alleles of Pom152-GFP (Table 2) and pKW1803 (HDEL-dsRed) linearized with EcoRV (Madrid et al., 2006). For fluorescence microscopy, cells were grown overnight at $24^{\circ} \mathrm{C}$ in SD-Leu media to early log phase $\left(\mathrm{A}_{600}=0.05-0.2\right)$ and visualized under direct fluorescence using a Nikon E400 microscope. KBY850 (nup 1334) strains were shifted to $35^{\circ} \mathrm{C}$ for $1 \mathrm{~h}$ prior to microscopy. Images were captured using an RTKE Spot CCD camera and SPOT-RTKe Imaging software (Diagnostic Instruments, Inc.). A one-way ANOVA was conducted to determine if the frequency of
Pom152 clustering at the nuclear envelope was different between the Pom152-GFP constructs tested in wt and in pom1524 yeast. In the wt yeast background, there was one outlier, as determined by boxplot, and the data failed to meet normality as assessed by Shapiro-Wilk test and equality of variances as assessed by Bartlett's test. To satisfy ANOVA assumptions, the outlier was removed, and the data were $\log$ transformed. Following data processing, a Shapiro-Wilk test $(P=0.23)$ and visual inspection of a histogram and a QQ plot revealed that the data were normally distributed. There was also equality of variances as assessed by Bartlett's test of homogeneity of variances $(P=0.77)$. A one-way ANOVA was performed on this transformed dataset. In pom152 1 yeast, a similar one-way ANOVA analysis was performed. The visual inspection of a histogram and a QQ plot and Shapiro-Wilk test confirmed normal distribution $(P=0.74)$. Bartlett's test confirmed homogeneity of variances $(P=0.14)$, and no outliers were detected using boxplot analysis.

\section{Crz1 nuclear protein export assays}

Kinetic analyses of Crz1-GFP nuclear export in wt and POM152 mutant strains were performed as described previously (Finn et al., 2013). Briefly, pLMB127 (Boustany and Cyert, 2002) was transformed into yeast strains BY4742, KBY776, KBY893, KBY1377, and KBY1378 to allow for Crz1GFP expression. Crzl-GFP was induced to accumulate in the nucleus by inclusion of $170 \mathrm{mM} \mathrm{CaCl}_{2}$ in SD-Leu media, and Crz1-GFP nuclear export was initiated by the addition of $1.5 \mu \mathrm{g} / \mathrm{ml} \mathrm{FK506.} \mathrm{The} \mathrm{percent} \mathrm{of} \mathrm{cells}$ exhibiting distinctly nuclear GFP fluorescence was calculated by direct observation immediately before FK506 addition $(\mathrm{t}=0)$ and at 2 min intervals after. Export assays were performed blind with each yeast strain assayed at least three times and greater than 100 cells observed at each timepoint. Robust regression analysis was performed using the Theil-Sen estimator (Sen, 1968). The Crz1 nuclear export data failed linear regression assumptions due to outliers and heteroscedasticity in the data. Outliers are checked with Cook's distance (Cook, 1977). A visual inspection of fitted values versus residuals indicated that the data does not satisfy homoscedasticity assumption. Thus, the Theil-Sen estimator (Sen, 1968) was used for regression analysis and the Mann-Whitney U-test for comparison of the resulting slopes. The $P$-value adjustment for multiple testing is performed with Benjamini-Hochberg method (Benjamini and Hochberg, 1995). 


\section{Acknowledgements}

The authors thank Martha Cyert, Karsten Weis, Munira Basrai, Pamela Silver, and Richard Wozniak for their sharing of yeast strains and plasmids, Karyn Belanger and Sue Geier for their expert technical contributions, organizational skills, and mentorship, and Ahmet Ay for his generous dedication of time and knowledge to the statistical analyses in this paper. We also thank undergraduate colleagues in the Belanger lab who provided valuable discussions and insights and especially acknowledge Caroline Adams, Nolan Sheppard, Sarah Kruse, Mohammed Rahman, lustin Moga, and Elizabeth Rivers for their work on Pom152-related projects.

\section{Competing interests}

The authors declare no competing or financial interests.

\section{Author contributions}

Conceptualization: K.D.B.; Methodology: J.T.B., A.J.H., C.M.W., K.D.B.; Validation: K.D.B.; Investigation: J.T.B., A.J.H., C.M.W., K.D.B.; Writing - original draft: J.T.B., K.D.B.; Writing - review \& editing: J.T.B., A.J.H., C.M.W., K.D.B.; Visualization J.T.B., A.J.H., K.D.B.; Supervision: K.D.B.; Project administration: K.D.B.; Funding acquisition: K.D.B.

\section{Funding}

This work was generously funded by National Institutes of Health (Grant R15 GM65107 to K.D.B.) and by Colgate University through the Faculty Research Council and the Stuart Updike Fund in support of undergraduate biology research.

\section{References}

Alber, F., Dokudovskaya, S., Veenhoff, L. M., Zhang, W., Kipper, J., Devos, D. Suprapto, A., Karni-Schmidt, O., Williams, R., Chait, B. T. et al. (2007a) Determining the architectures of macromolecular assemblies. Nature 450 683-694. doi:10.1038/nature06404

Alber, F., Dokudovskaya, S., Veenhoff, L. M., Zhang, W., Kipper, J., Devos, D. Suprapto, A., Karni-Schmidt, O., Williams, R., Chait, B. T. et al. (2007b). The molecular architecture of the nuclear pore complex. Nature 450, 695-701. doi:10.1038/nature06405

Altschul, S. F., Gish, W., Miller, W., Myers, E. W. and Lipman, D. J. (1990). Basic local alignment search tool. J. Mol. Biol. 215, 403-410. doi:10.1016/S00222836(05)80360-2

Beck, M. and Hurt, E. (2017). The nuclear pore complex: understanding its function through structural insight. Nat. Rev. Mol. Cell Biol. 18, 73-89. doi:10.1038/nrm. 2016.147

Belgareh, N. and Doye, V. (1997). Dynamics of nuclear pore distribution in nucleoporin mutant yeast cells. J. Cell Biol. 136, 747-759. doi:10.1083/jcb.136.4 747

Belanger, K. D., Gupta, A., MacDonald, K. M., Ott, C. M., Hodge, C. A., Cole, C. M. and Davis, L. I. (2005). Nuclear pore complex function in saccharomyces cerevisiae is influenced by glycosylation of the transmembrane nucleoporin Pom152p. Genetics 171, 935-947. doi:10.1534/genetics.104.036319

Benjamini, Y. and Hochberg, Y. (1995). Controlling the false discovery rate: a practical and powerful approach to multiple testing. J R. Stat. Soc. B (Methodological) 57, 289-300. doi:10.1111/j.2517-6161.1995.tb02031.x

Boustany, L. M. and Cyert, M. S. (2002). Calcineurin-dependent regulation of Crz1p nuclear export requires Msn5p and a conserved calcineurin docking site. Genes Dev. 16, 608-619. doi:10.1101/gad.967602

Cook, R. D. (1977). Detection of influential observation in linear regression. Technometrics 19, 15-18. doi:10.1080/00401706.1977.10489493

Finn, E. M., DeRoo, E. P., Clement, G. W., Rao, S., Kruse, S. E., Kokanovich, K. M. and Belanger, K. D. (2013). A subset of FG-nucleoporins is necessary for efficient Msn5-mediated nuclear protein export. Biochim Biophys. Acta 1833, 1096-1103. doi:10.1016/j.bbamcr.2012.12.020

Gietz, R. D. and Woods, R. A. (2006). Yeast transformation by the LiAc/SS Carrie DNA/PEG method. Methods Mol. Biol. 313, 107-120. doi:10.1385/1-59259-9583:107

Greber, U. F., Senior, A. and Gerace, L. (1990). A major glycoprotein of the nuclear pore complex is a membrane-spanning polypeptide with a large lumenal domain and a small cytoplasmic tail. EMBO J. 9, 1495-1502. doi:10.1002/j.1460-2075. 1990.tb08267.X

Hao, Q., Zhang, B., Yuan, K., Shi, H. and Blobel, G. (2018). Electron microscopy of Chaetomium Pom152 shows the assembly of ten-bead string. Cell Discov 4, 56. doi:10.1038/s41421-018-0057-7
Kim, S. J., Fernandez-Martinez, J., Nudelman, I., Shi, Y., Zhang, W., Raveh, B. Herricks, T., Slaughter, B. D., Hogan, J. A., Upla, P. et al. (2018). Integrative structure and functional anatomy of a nuclear pore complex. Nature 555, 475-482. doi:10.1038/nature26003

Knockenhauer, K. E. and Schwartz, T. U. (2016). The nuclear pore complex as a flexible and dynamic gate. Cell 164, 1162-1171. doi:10.1016/j.cell.2016.01.034

Kosinski, J., Mosalaganti, S., von Appen, A., Teimer, R., DiGuilio, A. L., Wan, W., Bui, K. H., Hagen, W. J. H., Briggs, J. A. G., Glavy, J. S. et al. (2016). Molecular architecture of the inner ring scaffold of the human nuclear pore complex. Science 352, 363-365. doi:10.1126/science.aaf0643

Lin, D. H., Stuwe, T., Schilbach, S., Rundlet, E. J., Perriches, T., Mobbs, G. Fan, Y., Thierbach, K., Huber, F. M., Collins, L. N. et al. (2016). Architecture of the symmetric core of the nuclear pore. Science 352, aaf1015. doi:10.1126/ science.aaf1015

Madrid, A. S., Mancuso, J., Cande, W. Z. and Weis, K. (2006). The role of the integral membrane nucleoporins Ndc1p and Pom152p in nuclear pore complex assembly and function. J. Cell Biol. 173, 361-371. doi:10.1083/jcb. 200506199

Makio, T., Stanton, L. H., Lin, C.-C., Goldfarb, D. S., Weis, K. and Wozniak, R. W. (2009). The nucleoporins Nup170p and Nup157p are essential for nuclear pore complex assembly. J. Cell Biol. 185, 459-473. doi:10.1083/jcb. 200810029

Marelli, M., Lusk, C. P., Chan, H., Aitchison, J. D. and Wozniak, R. W. (2001). A link between the synthesis of nucleoporins and the biogenesis of the nuclear envelope. J. Cell Biol. 153, 709-724. doi:10.1083/jcb.153.4.709

Mi, L., Goryaynov, A., Lindquist, A., Rexach, M. and Yang, W. (2015). Quantifying nucleoporin stoichiometry inside single nuclear pore complexes In vivo. Sci. Rep. 5, 9372. doi:10.1038/srep09372

Onischenko, E. and Weis, K. (2011). Nuclear pore complex-a coat specifically tailored for the nuclear envelope. Curr. Opin. Cell Biol. 23, 293-301. doi:10.1016/j. ceb.2011.01.002

Onischenko, E., Stanton, L. H., Madrid, A. S., Kieselbach, T. and Weis, K. (2009). Role of the Ndc1 interaction network in yeast nuclear pore complex assembly and maintenance. J. Cell Biol. 185, 475-491. doi:10.1083/jcb 200810030

Rajoo, S., Vallotton, P., Onischenko, E. and Weis, K. (2018). Stoichiometry and compositional plasticity of the yeast nuclear pore complex revealed by quantitative fluorescence microscopy. Proc. Natl. Acad. Sci. USA 115, E3969-E3977. doi:10.1073/pnas.1719398115

Rout, M. P., Aitchison, J. D., Suprapto, A., Hjertaas, K., Zhao, Y. and Chait, B. T. (2000). The yeast nuclear pore complex: composition, architecture, and transport mechanism. J. Cell Biol. 148, 635-651. doi:10.1083/jcb.148.4.635

Sen, P. K. (1968). Estimates of the regression coefficient based on Kendall's tau. J. Am. Stat. Assoc. 63, 1379-1389. doi:10.1080/01621459.1968. 10480934

Sikorski, R. S. and Hieter, P. (1989). A system of shuttle vectors and yeast host strains designed for efficient manipulation of DNA in Saccharomyces cerevisiae. Genetics 122, 19-27. doi:10.1093/genetics/122.1.19

Stavru, F., Nautrup-Pedersen, G., Cordes, V. C. and Görlich, D. (2006). Nuclea pore complex assembly and maintenance in POM121- and gp210-deficient cells. J. Cell Biol. 173, 477-483. doi:10.1083/jcb.200601002

Tcheperegine, S. E., Marelli, M. and Wozniak, R. W. (1999). Topology and functional domains of the yeast pore membrane protein Pom152p. J. Biol. Chem. 274, 5252-5258. doi:10.1074/jbc.274.8.5252

Tong, A. H. Y. and Boone, C. (2006). Synthetic genetic array analysis in Saccharomyces cerevisiae. Methods Mol. Biol. 313, 171-192. doi:10.1385/159259-958-3:171

Tran, E. J. and Wente, S. R. (2006). Dynamic nuclear pore complexes: life on the edge. Cell 125, 1041-1053. doi:10.1016/j.cell.2006.05.027

Upla, P., Kim, S. J., Sampathkumar, P., Dutta, K., Cahill, S. M., Chemmama, I. E., Williams, R., Bonanno, J. B., Rice, W. J., Stokes, D. L. et al. (2017). Molecula architecture of the major membrane ring component of the nuclear pore complex. Structure 25, 434-445. doi:10.1016/j.str.2017.01.006

Wozniak, R. W. and Blobel, G. (1992). The single transmembrane segment of gp210 is sufficient for sorting to the pore membrane domain of the nuclea envelope. J. Cell Biol. 119, 1441-1449. doi:10.1083/jcb.119.6.1441

Wozniak, R. W., Blobel, G. and Rout, M. P. (1994). POM152 is an integral protein of the pore membrane domain of the yeast nuclear envelope. J. Cell Biol. 125, 31-42. doi:10.1083/jcb.125.1.31

Yewdell, W. T., Colombi, P., Makhnevych, T. and Lusk, C. P. (2011). Lumena interactions in nuclear pore complex assembly and stability. Mol. Biol. Cell 22 1375-1388. doi:10.1091/mbc.e10-06-0554 